\title{
The Nature of the Spectrum for a Landau Hamiltonian with Delta Impurities
}

\author{
T. C. Dorlas, ${ }^{1}$ N. Macris, ${ }^{2}$ and J. V. Pulé 3.4
}

Received August 6, 1996

\begin{abstract}
We consider a single-band approximation to the random Schrödinger operator in an external magnetic field. The random potential consists of delta functions of random strengths situated on the sites of a regular two-dimensional lattice. We characterize the entire spectrum of this Hamiltonian when the magnetic field is sufficiently high. We show that the whole spectrum is pure point, the energy coinciding with the first Landau level in the absence of a random potential being infinitely degenerate, while the eigenfunctions corresponding to energies in the rest of the spectrum are localized.
\end{abstract}

KEY WORDS: Quantum Hall eflect: Landau Hamiltonian; localization; localization length: delta impurities.

\section{INTRODUCTION}

The study of random Schrödinger operators with magnetic fields is important for the theory of the quantum Hall effect and is also of intrinsic mathematical interest. A basic picture that is widely used in the theories of the quantum Hall effect is the following. In each Landau band, all eigenenergies except for one energy, $E_{c}$ say, in the center of the band, correspond to exponentially localized wave functions and the localization length $\xi(E)$ diverges like $\left|E-E_{c}\right|^{-1}$, with $v>0$, at $E_{c}$. This picture is supported

\footnotetext{
' Department of Mathematics, University College of Swansea, Singleton Park, Swansea SA2 8PP, Wales, U.K.; e-mail: T.C.Dorlas $a$, swansea.ac.uk.

2Institut de Physique Theorique, Ecole Polytechnique Fédérale de Lausanne, CH 1015 Lausanne. Switzerland; e-mail: macris $a$ eldp.epfl.ch.

"Department of Mathematical Physics, University College. Belfield, Dublin 4, Ireland: e-mail: jpule $(a$ acadamh.ucd.ie.

${ }^{4}$ Research Associate, School of Theoretical Physics, Dublin Institute for Advanced Studies. Dublin, lreland.
} 
by field-theoretic approaches and also by the so-called network model. Much effort has been devoted to the numerical calculation of the exponent, and at least for models restricted to the first Landau band, it is found that $v \approx 2.3$, in agreement with experiment, quite independently of the microscopic details of the randomness. We refer to the recent review ${ }^{(1)}$ for more information on these matters.

Mathematical proofs that the wavefunctions are localized, and thus the spectrum is pure point, at the edges of the Landau bands have been achieved recently. ${ }^{(2-6)}$ However very little is known about the nature of the spectrum in the interior of the band. Avishai et al. ${ }^{(7)}$ have shown that in the model studied in this paper there is an extended generalized eigenfunction at the first Landau energy.

In earlier works ${ }^{\left({ }^{8.9}\right)}$ it was assumed that there exist localized states at the band edges. With this assumption it was deduced from the fact that the Hall conductivity is nonvanishing that if all states are finitely degenerate, then the localization length diverges somewhere in the middle of the band. However, there are various possibilities for the spectrum which are compatible with this statement. One possibility is a continuous spectrum, but it was already suggested by Thouless ${ }^{(10)}$ that the spectrum in the center of the band might be singular. In this paper we prove, for the model considered, that the situation is in fact as follows. The whole spectrum is pure point, but there is a single eigenvalue, corresponding to the first Landau level, which is infinitely degenerate. We do not prove exponential decay, but the decay that we obtain is sufficient to show that the eigenstates other than the ones corresponding to the Landau levels do not contribute to the conductivity and thus that the conductivity is entirely due to the infinite degeneracy.

We consider a two-dimensional infinite system of noninteracting electrons moving in a uniform magnetic field of strength $B$ and a random potential $V$. In the symetric gauge the vector potential is given by $A(x)=$ $\left(-\frac{1}{2} B x_{2}, \frac{1}{2} B x_{1}\right), x=\left(x_{1}, x_{2}\right) \in \mathbb{R}^{2}$, and the Hamiltonian is

$$
H=(-i \nabla-A(x))^{2}+V(x)
$$

The effect of the random potential is to broaden the Landau levels into bands. When the potential is not too strong compared to the magnetic field, these bands do not overlap. The random potential consists of point scatterers, delta functions, situated on the sites of a regular lattice. The strengths of the scatterers are random; they are independent, identically distributed variables with a bounded probability distribution. The precise hypotheses on the probability distribution are stated in Section 2. When the magnetic field is strong, it is reasonable to consider only the projections 
of the Hamiltonian onto each Landau level and to neglect the cross terms. The Hamiltonian restricted to the $n$th level is

$$
H_{n}=B(2 n+1) P_{n}+P_{n} V P_{n}
$$

where $P_{n}$ denotes the projection onto the level. The term $B(2 n+1) P_{n}$ comes from the decomposition of the purely kinetic part of (1.1) and can be dropped, as it modifies the energy only by a constant. Note that the resulting Hamiltonian is a random integral operator instead of a differential operator and that the kernels of $P_{n}$ are known explicitly. For simplicity, in this paper we restrict ourselves to the case $n=0$, but the case $n \neq 0$ can be treated similarly.

This model has been the subject of many numerical calculations, leading, among other things, to the exponent $v^{(1.11 .12)}$ (In refs. 11 and 12 the strength takes only two values $\pm V$.) Our aim is to investigate the validity of the physical picture outlined in the first paragraph.

The results obtained in this paper are valid in the regime when the magnetic field is large compared to the density of scatterers. We prove that there exists an infinitely degenerate eigenenergy which coincides with the first Landau level in the absence of a random potential. This is implied in the work of Avishai et al. ${ }^{(7)}$ But the main contribution of this paper is to show that the whole spectrum is pure point and to prove that the eigenfunctions corresponding to energies not coinciding with the first Landau level are localized. Our estimate on the localized wavefunctions show, that for every $0<\gamma<1$, if the magnetic field is sufficiently high depending on $\gamma$, they decay faster than $\exp \left(-m|x|^{\prime}\right)$ for all $m>0$, independent of the energy. Note that this result does not exclude exponential decay nor the possibility that the (exponential) localization length diverges.

We expect that at least in the strong magnetic field regime considered here the results will be unchanged in a more general model where the Hamiltonian is not restricted to the first Landau band and the position of the scatterers is random.

The paper is organized as follows. In Section 2 we set up precisely the model Hamiltonian and study the eigenspace corresponding to the lowest Landau level. The proof that the spectrum is pure point for all energies relies on the general theorem of von Dreifus and Klein. ${ }^{(13)}$ In Section 3 we prove the two basic hypotheses of this theorem for all energies: (i) that with high probability the Green function decays in some large box; and (ii) the nonresonance condition. In ref. 4 we adapted the von Dreifus and Klein theorem to the magnetic continuum case for another type of random potential. The necessary modifications needed here are briefly sketched in Section 4. 


\section{THE HAMILTONIAN AND ITS NULL SPACE}

Let $\omega_{n}, n \in \mathbb{Z}[i] \equiv\left\{n_{1}+i n_{2}:\left(n_{1}, n_{2}\right) \in \mathbb{Z}^{2}\right\}$, the Gaussian integers, be i.i.d. random variables. We shall assume that their distribution is given by an absolutely continuous probability measure $\mu$ whose support is a compact interval $X=[a, b] \subset \mathbb{R}$ containing the origin and whose density $\rho$ is bounded. We let $\Omega=X^{\mathbb{Z}[i]}$ and $\mathbb{P}=\prod_{n \in \mathbb{Z}[i]} \mu$. For $m \in \mathbb{Z}[i]$ let $\tau_{m}$ be the measure-preserving automorphism of $\Omega$ defined by

$$
\left(\tau_{m} \omega\right)_{n}=\omega_{n-m}
$$

The group $\left\{\tau_{m}: m \in \mathbb{Z}[i]\right\}$ is ergodic for the probability measure $\mathbb{P}$.

Let $\mathscr{H}=L^{2}(\mathbb{C})$ and let $\mathscr{H}_{0}$ be the eigenspace corresponding to the lowest eigenvalue (first Landau level) of the kinetic part of the Hamiltonian defined in (1.1) and let $P_{0}$ be the orthogonal projection onto $\mathscr{H}_{0}$. The Hamiltonian for our model is the operator on $\mathscr{H}_{0}$ given formally by

$$
H(\omega)=\frac{\pi}{2 \kappa} P_{0} V(\cdot, \omega)=\frac{\pi}{2 \kappa} P_{0} V(\cdot, \omega) P_{0}
$$

where $\kappa=B / 4, \omega \in \Omega$, and

$$
V(z, \omega)=\sum_{n \in \mathbb{Z}[i]} \omega_{n} \delta(z-n)
$$

Note that $H$ coincides with $H_{0}$ in (1.2) up to the term $B P_{0}$ and a multiplicative constant. The projection $P_{0}$ is an integral operator with kernel

$$
P_{0}\left(z, z^{\prime}\right)=\frac{2 \kappa}{\pi} \exp \left[-\kappa\left|z-z^{\prime}\right|^{2}-2 i \kappa z \wedge z^{\prime}\right]
$$

where $z \wedge z^{\prime}=\mathscr{R} z \mathscr{I} z^{\prime}-\mathscr{I} z \mathscr{R} z^{\prime}, \mathscr{R} z$ and $\mathscr{I} z$ being the real and imaginary parts of $z$, respectively. Note that if $\psi \in \mathscr{H}$, then $\psi \in \mathscr{H}_{0}$ if and only if $\psi(z)=f(z) \exp \left(-\left.\kappa\right|^{2}\right)$ where $f(z)$ is entire. Using (2.4), we can write the Hamiltonian in the form

$$
H=\sum_{n \in \mathbb{Z}[i]} \omega_{n} f_{n} \otimes \overline{f_{n}}
$$

where, for $n \in \mathbb{Z}[i]$,

$$
f_{n}(z)=\sqrt{\frac{\pi}{2 \kappa}} P_{0}(z, n)=\sqrt{\frac{2 \kappa}{\pi}} \exp \left[2 \kappa \bar{n} z-\kappa\left(|n|^{2}+|z|^{2}\right)\right]
$$


Note that $\left\|f_{n}\right\|=1,\left(f_{n}, f_{m}\right)=(\pi / 2 \kappa)^{1 / 2} f_{m}(n)$ and that $H$ is an integral operator with kernel

$$
H\left(z, z^{\prime}\right)=\sum_{n \in \mathbb{Z}[i]} \omega_{n} f_{n}(z) \overline{f_{n}\left(z^{\prime}\right)}
$$

We first obtain a bound on $H\left(z, z^{\prime}\right)$ which implies that $H$ is bounded. The following is proved in the Appendix.

Lemma 2.1. For $s, t>0$ and $z, z^{\prime} \in \mathbb{C}$

$$
\sum_{n \in \mathbb{Z}[i]} e^{-s|=-n|^{2}} e^{-t\left|n-z^{\prime}\right|^{2}} \leqslant K(s+t) e^{-[s t / s+t)]\left|=-z^{\prime}\right|^{2}}
$$

where

$$
K(s)=\left(1+e^{-s / 4}+\left(\frac{\pi}{s}\right)^{1 / 2}\right)^{2}
$$

The above lemma implies that $\left|H\left(z, z^{\prime}\right)\right|$ is bounded above by

$$
\frac{2 M \kappa}{\pi} K(2 \kappa) e^{-(\kappa / 2)\left|=-z^{\prime}\right|^{2}}
$$

where $M=\max (|a|,|b|)$. Therefore $H$ is bounded and $\|H\| \leqslant 4 M K(2 \kappa)$. Note that the heat kernel is

$$
P_{,}\left(z, z^{\prime}\right)=\frac{1}{2 \pi t} e^{-(1 / 2 t) 1=-\left.z^{\prime}\right|^{2}}
$$

and the corresponding operator has unit norm.

Let $\left\{U_{z}: z \in \mathbb{C}\right\}$ be the family of unitary operators on $\mathscr{H}$ corresponding to the magnetic translations:

$$
\left(U_{z} f\right)\left(z^{\prime}\right)=e^{2 i \kappa z} \wedge^{\prime} z^{\prime} f\left(z+z^{\prime}\right)
$$

Then for $n \in \mathbb{Z}[i]$

$$
U_{n} H(\omega) U_{n}^{-1}=H\left(\tau_{n} \omega\right)
$$

Note that $\left[P_{0}, U_{z}\right]=0$ for all $z \in \mathbb{C}$, so that $U_{z} \mathscr{H}_{0} \subset \mathscr{H}_{0}$. Also $U_{z 1} U_{z 2}=$ $\left[\exp \left(2 i \kappa z_{2} \wedge z_{1}\right)\right] U_{z_{1}+z_{2}}$. The ergodicity of $\left\{\tau_{m}: m \in \mathbb{Z}[i]\right\}$ and Eq. (2.9) together imply that the spectrum of $H(\omega)$ and its components are nonrandom (see, for example, ref. 14, Theorem V.2.4); it is easy to prove that almost surely the spectrum of $H(\omega)$ contains $X$ : 
Lemma 2.2. With probability one

$$
X \subset \sigma(H(\omega))
$$

Proof. It is sufficient to prove that for each $E \in[a, b]$ and for all $\delta>0$, there exists $\Omega^{\prime}$ with $\mathbb{P}\left(\Omega^{\prime}\right)>0$ and $\psi \in \mathscr{H}_{0}$ with $\|\psi\|=1$ such that for all $\omega \in \Omega^{\prime},\|(H(\omega)-E) \psi\|<\delta$. Choose $R$ such that $\sum_{|m| \geqslant R} \exp \left(-\kappa|n|^{2}\right)<$ $\delta / 3 M$, where $M=\max (|a|,|b|)$, and let

$$
\Omega^{\prime}=\left\{\omega:\left|\omega_{0}-E\right|<\delta / 3, \max _{|m|<R . m \neq 0}\left|\omega_{m}\right| K(\kappa)<\delta / 3\right\}
$$

Since $E$ and 0 are in the support of $\mu, \mathbb{P}\left(\Omega^{\prime}\right)>0$. We have

$$
\begin{aligned}
\left(H f_{0}-E f_{0}\right)(z) & =\sqrt{\frac{\pi}{2 \kappa}} \sum_{n} \omega_{n} f_{n}(z) f_{0}(n)-E f_{0}(z) \\
& =\left(\omega_{0}-E\right) f_{0}(z)+\sqrt{\frac{\pi}{2 \kappa}} \sum_{n \neq 0} \omega_{n} f_{n}(z) f_{0}(n)
\end{aligned}
$$

Therefore, since $\sum_{n \in \mathbb{Z}[i]} \exp \left(-\kappa^{-}|n|^{2}\right) \leqslant K(\kappa)$, for $\omega \in \Omega^{\prime}$,

$$
\begin{gathered}
\left\|H f_{0}-E f_{0}\right\| \leqslant \\
+M \sqrt{\frac{\pi}{2 \kappa}} \sum_{\mid \geqslant R}\left|f_{0}(n)\right|<\delta\left|+\sqrt{\frac{\pi}{2 k}} \sum_{\substack{n \neq 0 \\
|n|<R}}\right| \omega_{n}|\cdot| f_{0}(n) \mid \\
+M
\end{gathered}
$$

In the rest of this section we examine the null space of $H, N(H)$. We characterize it and show that it is infinite dimensional for large $\kappa$. Let $\mathscr{H}_{1}$ be the closure of the span of $\left\{f_{n}: n \in \mathbb{Z}[i]\right\}$ and $\mathscr{M}_{0}=\mathscr{H}_{1}^{\perp}$. Clearly $\mathscr{H}_{0} \subset \mathscr{H}(H)$. We shall prove that $\mathscr{H}_{0}=\mathscr{H}(H)$ and that $\mathscr{H}_{0}$ is infinite dimensional.

Lemma 2.3. There exists $\kappa_{0}>0$ such that for $k>\kappa_{0}$, with probability one, $\mathscr{H}_{0}=\mathscr{N}(H)$ and $\mathscr{H}_{0}$ is infinite dimensional.

Proof. If $\psi \in \mathscr{H}_{0}$, then for all $n \in \mathbb{Z}[i], \psi(n)=\left(P_{0} \psi\right)(n)=(2 \kappa / \pi)^{1 / 2}$ $\left(f_{n}, \psi\right)$. Therefore $\psi \in \mathscr{A}_{0}$ if and only if for all $n \in \mathbb{Z}[i], \psi(n)=0$. Suppose $H \psi=0$; we want to show that $\psi(n)=0$ for all $n \in \mathbb{Z}[i]$. We have

$$
H \psi=\sum_{n \in \mathbb{Z}[i]} \alpha_{n} f_{n}
$$


where

$$
\alpha_{n}=\sqrt{\frac{\pi}{2 \kappa}} \omega_{n} \psi(n)
$$

Thus

$$
\begin{aligned}
\|H \psi\|^{2} & =\lim _{R \rightarrow \infty}\left(\sum_{|m|<R}\left|\alpha_{n}\right|^{2}+\sum_{\substack{|,|<R \\
|m|<<\\
n \neq m}} \bar{\alpha}_{n} \alpha_{m}\left(f_{n}, f_{m}\right)\right) \\
& \geqslant \lim _{R \rightarrow \infty}\left(2 \sum_{\substack{|m|<R \\
|m|<}}\left|\alpha_{n}\right|^{2}-\sum_{\substack{|m|<R \\
|m|<R}}\left|\alpha_{n}\right| \cdot\left|\alpha_{m}\right| e^{-\kappa|| m-\left.m\right|^{2}}\right)
\end{aligned}
$$

Now

$$
\begin{aligned}
& \sum_{\substack{|m|<R \\
|m|<R}}\left|\alpha_{n}\right| \cdot\left|\alpha_{m}\right| e^{-\kappa|n-m|^{2}} \\
& \quad=\sum_{r \in \mathbb{Z}[i]} e^{-\kappa|r|^{2}} \sum_{\substack{|,|<|<R\\
| n+r \mid<R}}\left|\alpha_{n}\right| \cdot\left|\alpha_{n+r}\right| \leqslant K(\kappa) \sum_{|m|<R}\left|\alpha_{n}\right|^{2}
\end{aligned}
$$

Thus

$$
0=\|H \psi\|^{2} \geqslant(2-K(\kappa)) \sum_{n \in \mathbb{Z}[i]}\left|\alpha_{n}\right|^{2}
$$

and so if $\kappa$ is large enough, then $\sum_{n \in \mathbb{Z}[i]}\left|\alpha_{n}\right|^{2}=0$. Since almost surely $\omega_{n} \neq 0$ for all $n \in \mathbb{Z}[i], \psi(n)=0$ for all $n \in \mathbb{Z}[i]$, with probability one. Thus we have proved that $\mathscr{H}_{0}=\mathscr{N}(H)$ almost surely. Let

$$
\psi_{0}(z)=\prod_{n \neq 0}\left(1-\frac{z}{n}\right) e^{z / n+z^{2} / 2 n^{2}}
$$

It follows from the theory of entire functions (see ref. 15, 2.10.1) that there exists $A>0$ such that $\left|\psi_{0}(z)\right| \leqslant \exp \left(A|z|^{2}\right)$. Let $\phi_{k}(z)=z^{k} \psi_{0}(z)$ $\exp \left(-k|z|^{2}\right)$ for $k \geqslant 1$; then if $k>A$, the $\phi_{k}$ are in $\mathscr{M}_{0}$. Moreover if $\sum_{j=1}^{N} a_{k j} \phi_{k_{j}}=0$, then $\sum_{j=1}^{N} a_{k_{j}} z^{k_{j}}=0$ for $z \notin \mathbb{Z}[i]$. Therefore, $\sum_{j=1}^{N} a_{k_{j}} z^{k_{j}} \equiv 0$ and thus the $a_{k,}$ are zero, implying that the $\phi_{k}$ are independent.

Remarks. (i) There exists $\kappa_{1}>0$ such that $\sup _{|=|=R}\left|\psi_{0}(z)\right| \geqslant$ $\exp \left(\kappa_{1} R^{2}\right)$ (cf. ref. $\left.15,2.5 .11\right)$. 
(ii) It is possible to give a characterization of the elements of $\mathscr{M}_{0}{ }^{\left({ }^{7)}\right.}$ If $\psi \in \mathscr{M}_{0}$, then $\psi(z)=f(z) \phi(z) \exp \left(-\kappa|z|^{2}\right)$, where $f$ and $\phi$ are entire and $\phi$ has simple zeros at all $n \in \mathbb{Z}[i]$ and no other zeros. Then, since, $\sum_{n \neq 0}\left(1 /|n|^{3}\right)$ converges by Hadamard's factorization theorem (see ref. 15, 2.7.1) there is an entire function $h$ such that

$$
\phi(z)=z e^{n z=)} \psi_{0}(z)
$$

Therefore

$$
\psi(z)=f(z) z e^{h(z)} \psi_{0}(z) e^{-\kappa^{*} \mid z^{2}}=z g(z) \psi_{0}(z) e^{-\kappa^{*}|=|^{2}}
$$

where $g(z)$ is entire.

(iii) We feel that we should make some comments about a paper by Thouless ${ }^{(16)}$ on the Wannier functions. Thouless pointed out that if $\alpha_{n}=$

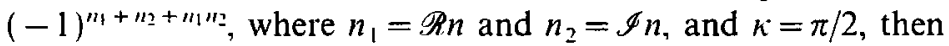

$$
\sum_{n \in \mathbb{Z}[i]} \alpha_{n} f_{n}(z) \equiv 0
$$

We first note that this does not contradict Lemma 2.3, since our result holds only for large $\kappa$. Also, it does not imply that the $f_{n}$ are not linearly independent even for $\kappa=\pi / 2$, that is, if $\sum_{j=1}^{N} \alpha_{j} f_{n}(z) \equiv 0$ with $N<\infty$, then $\alpha_{j}=0$ for $j=1, \ldots, N$, as can be seen from the following argument. We have

$$
0=\sum_{j=1}^{N} \alpha_{j} f_{n_{j}}(z)=\left(\sum_{k=0}^{\infty} c_{k} z^{k}\right) e^{-k|=|^{2}}
$$

where

$$
c_{k}=\frac{(2 \kappa)^{k}}{k !} \sum_{j=1}^{N} \alpha_{j} \bar{n}_{j}^{k} e^{-\kappa \cdot\left|m_{j}\right|^{2}}=\frac{(2 \kappa)^{k}}{k !} \sum_{j=1}^{N} \bar{a}_{j} \bar{n}_{j}^{k}
$$

with

$$
a_{j}=\bar{\alpha}_{j} e^{-n^{n}\left|n_{j}\right|^{2}}
$$

Let $\psi_{0}(z)$ be as in (2.13) and for $n^{\prime} \in \mathbb{Z}[i], n^{\prime} \neq 0$, let

$$
\psi_{n^{\prime}}(z)=z \prod_{n \neq 0, n^{\prime}}\left(1-\frac{z}{n}\right) e^{z / n+z^{2} / 2 n^{2}}
$$


The functions $\psi_{n^{\prime}}(z)$ are entire and

$$
\psi_{n^{\prime}}(n)\left\{\begin{array}{lll}
=0 & \text { if } & n \neq n^{\prime} \\
\neq 0 & \text { if } \quad n=n^{\prime}
\end{array}\right.
$$

Let

$$
\psi_{n^{\prime}}(z)=\sum_{k=0}^{\infty} b_{n \prime^{\prime} z^{k}} z^{k}
$$

Since $\sum_{j=1}^{N} a_{j} n_{j}^{k}=0$ for $k=0,1,2, \ldots$, we have for $l=1, \ldots, N$,

$$
a_{,} \psi_{m !}\left(n_{i}\right)=\sum_{j=1}^{N} a_{j} \psi_{m}\left(n_{j}\right)=\sum_{k=0}^{\infty} b_{m k} \sum_{j=1}^{N} a_{j} n_{j}^{k}=0
$$

and therefore $\alpha_{1}=0$ for all $l=1, \ldots, N$.

\section{THE SPECTRUM OF $\boldsymbol{H}$}

In this section we prove that the whole spectrum of $H$ is pure point with probability one. We prove also that almost surely the eigenfunctions corresponding to nonzero eigenvalues are localized in the sense that they decay like $\exp \left(-|z|^{\gamma}\right)$ with $0<\gamma<1$. To do this we use the decomposition $\mathscr{H}_{0}=\mathscr{M}_{0} \oplus \mathscr{M}_{1}$ to write $H$ in the form

$$
H=0 \oplus H_{\mathrm{I}}
$$

where $H_{1}$ is the restriction of $H$ to $\mathscr{H}_{1}$, and then study the spectrum of $H_{1}$. We have that

$$
H_{1}=\sum_{n \in \mathbb{Z}[i]} \omega_{n} f_{n} \otimes \bar{f}_{n}
$$

The main result of this section is the following theorem.

Theorem 3.1. Let $0<\gamma<1$. There exists $\kappa_{1}(\gamma)$ such that for $\kappa>\kappa_{1}(\gamma)$, almost surely, the spectrum of $H_{1}$ is pure point and the generalized eigenfunctions of $H_{1}$ satisfy

$$
\lim _{z \rightarrow \infty} \frac{\ln |\psi(z)|}{|z|^{\gamma}}=-\infty
$$

The generalized eigenfunctions of $H_{1}$ are elements of a conuclear space $\mathscr{E}_{1}$ containing $\mathscr{M}_{1}$, which will be defined after Lemma 3.7. To prove this 
theorem we use the method of ref. 13, but we also exploit the fact that for large $\kappa, H_{1}$ is almost diagonal since the inner products $\left|\left(f_{n}, f_{m}\right)\right| \leqslant$ $\exp \left(-k^{2}|n-m|^{2}\right)$. For this purpose we define an orthonormal basis spanning $\mathscr{M}_{1}$ which approximates the set of $f_{11}$ for large $\kappa$. Let $\lambda: \mathbb{Z}[i] \rightarrow \mathbb{N}$ be any ordering of the elements of $\mathbb{Z}[i]$. Let $\left\{g_{n}: n \in \mathbb{Z}[i]\right\}$ be the orthonormal basis of $\mathscr{M}_{1}$ obtained by the Gram-Schmidt procedure:

$$
\begin{aligned}
& g_{0}=f_{0} \\
& g_{n}=D_{n}^{-1 / 2}\left\{f_{n}-\sum_{\lambda(m)<\lambda(n)}\left(g_{m}, f_{n}\right) g_{m}\right\}
\end{aligned}
$$

where

$$
D_{n}=1-\sum_{i(m)<i(n)}\left|\left(g_{m}, f_{n}\right)\right|^{2}
$$

From now on, for any operator $A$, we shall write $\langle n|A| m\rangle$ for the inner product $\left(g_{a}, A g_{m}\right)$. If $A$ is a subset of $\mathbb{Z}[i]$, let

$$
\hat{H}_{.1}=\sum_{n \in A} \omega_{n} f_{n} \otimes \bar{f}_{n}
$$

Let $P_{.}$be the projection onto the space spanned by $\left\{g_{n}: n \in A\right\}$, that is,

$$
P_{.1}=\sum_{n \in A} g_{n} \otimes \bar{g}_{n}
$$

and let

$$
H_{A}=P_{.1} \hat{H}_{.1} P_{A}=P_{.1} \sum_{n \in A} \omega_{n} f_{n} \otimes \bar{f}_{n} P_{A}
$$

and

$$
G_{.1}(E)=\left(E-H_{.4}\right)^{-1}
$$

Let us assume that there exist $0<\gamma^{\prime}<1, A>0$, and $R>0$ such that for all $n, m \in \mathbb{Z}[i]$,

$$
\begin{gathered}
\left|\left\langle n\left|\hat{H}_{A}\right| m\right\rangle\right| \leqslant A e^{-R|n-m| r^{2}} \\
\left\|\hat{H}_{A}\right\| \leqslant A
\end{gathered}
$$


and

$$
\left|\left\langle n\left|\hat{H}_{,}\right| m\right\rangle\right| \leqslant A e^{-R\left(d\left(n, A^{\prime}\right)+\left(t m . A^{\prime}\right)\right.}
$$

for all $A \subset \mathbb{Z}[i]$. These bounds will be proved in Lemma 3.7. We now fix numbers $s, \gamma_{0}, \beta, p$, and $q$ satisfying $1 / 2<s<1,0<\gamma_{0}<\gamma s, 0<\beta<\gamma_{0} s$, $p>2$, and $q>4 p+12$. These are fixed throughout the rest of the paper. We need the following definitions to state the main theorem, which is used in the proof of Theorem 3.1.

For $n \in \mathbb{Z}[i]$ let

$$
A_{L}(n)=\{m: m \in \mathbb{Z}[i],|\mathscr{R}(n-m)|<L / 2,|\mathscr{F}(n-m)|<L / 2\}
$$

Definition. If $r>0$ and $E \in \mathbb{R}$, we shall say that a square $A_{L}(n)$, with $n \in \mathbb{Z}[i]$, is $(r, E)$-regular if:

(RA) $d\left(E, \sigma\left(H_{t,(n)}\right)\right)>\frac{1}{2} e^{-L^{\prime \prime}}$

(RB) We have

$$
\left|\left\langle n\left|G_{A,(n)}(E)\right| m\right\rangle\right|<e^{-r L^{* * 1}}
$$

for all $m \in \partial \Lambda_{L}(n) \cap \mathbb{Z}[i]$, where $\partial \Lambda_{L}(n)=\Lambda_{L}(n) \backslash \Lambda_{\tilde{L}}(n)$ and $\tilde{L}=L-L^{*}$.

If $A_{L}(n)$ is not $(r, E)$-regular, we shall say that $A_{L}(n)$ is $(r, E)$-singular.

We shall say that the condition (PI) is satisfied if:

(P1) There exists $\eta>0$ such that, for all $n \in \mathbb{Z}[i]$, for all $L \geqslant L_{0}$ and all $E \in\left(E_{0}-\eta, E_{0}+\eta\right)$, we have

$$
\mathbb{P}\left\{d\left(E, \sigma\left(H_{L_{L}(1)}\right)\right)<e^{-L^{\prime \prime}}\right\}<L^{-q}
$$

and we shall say that the condition (P2) is satisfied if:

(P2) There exists $r>0$ such that, for all $n \in \mathbb{Z}[i]$,

$$
\mathbb{P}\left\{\Lambda_{L_{11}}(n) \text { is }\left(r, E_{0}\right) \text {-regular }\right\} \geqslant 1-L_{0}^{-p}
$$

Under assumption (3.9) the following theorem is proved in Section 4.

Theorem 3.2. There exists $L_{1}\left(s, \gamma, \gamma_{0}, \beta, p, q, A, R\right)$ such that if for some $E_{0} \in \mathbb{R}$ and for some $L_{0}>L_{1}$ both conditions (P1) and (P2) above are satisfied, then there is a $\Delta>0$ so that almost surely $\sigma\left(H_{1}\right) \cap$ $\left(E_{0}-\Delta, E_{0}+\Delta\right)$ is in the pure point spectrum and if $\psi$ is an eigenfunction of $H_{1}$ with eigenvalue in $\left(E_{0}-\Delta, E_{0}+\Delta\right)$, then for all $0<\gamma^{\prime}<\gamma_{0}$

$$
\lim _{z \rightarrow \infty} \frac{\ln |\psi(z)|}{|z|^{\prime}}=-\infty
$$


We shall now proceed to check that the conditions of the theorem are satisfied for all $E_{0} \in \mathbb{R}$, for all $0<\gamma<1,1 / 2<s<1,0<\gamma_{0}<\gamma s, 0<\beta<\gamma_{0} s$, with $p=3$ and $q=24$, when $\kappa$ is sufficiently large, depending on all these numbers except $E_{0}$. Theorem 3.1 then follows. First we need to establish some properties of the $g_{n}$. For the proof of the next lemma, see the Appendix.

Lemma 3.3. For $\gamma \in(0,1)$, there exists $C_{0}(\gamma)>0$ such that for $\alpha>1$

$$
\sum_{m \in \mathbb{Z}[i]} e^{-x|1=-m|^{i}+\left|z^{\prime}-m\right|^{\prime \prime}} \leqslant C_{0}(\gamma) e^{-x\left|z-z^{\prime}\right| i}
$$

We now use the preceding result to obtain bounds on the coefficients in the expansion of the $g_{n}$ in terms of the $f_{n}$.

Lemma 3.4. For $0<\gamma^{\prime}<1$, there exists $\kappa(\gamma)$ such that for all $\kappa>k(\gamma)$ and all $n \in \mathbb{Z}[i]$,

$$
g_{n}=\sum_{\lambda(m) \leqslant \lambda(n)} c_{m}^{\prime \prime} e^{-(\kappa / 4)|n-m| i} f_{m}
$$

where $\left|c_{m}^{\prime \prime}\right| \leqslant 1$ for $\lambda(m)<\lambda(n)$ and $1 \leqslant c_{n}^{\prime \prime} \leqslant 1+e^{-n / 8}$.

Proof. We use induction on $\lambda(n)$. By the induction hypothesis, if $\lambda(m) \leqslant \lambda(n)$, then

$$
\left(g_{m}, f_{n^{\prime}}\right)=\sum_{\lambda(u) \leqslant \lambda(m)} \bar{c}_{\prime \prime \prime} e^{-(\kappa / 4)|m-u|^{\prime \prime}}\left(f_{u}, f_{\prime^{\prime}}\right)
$$

Thus

$$
\begin{aligned}
& \sum_{\lambda(m) \leqslant \lambda(m)}\left(g_{m}, f_{n^{\prime}}\right) g_{m}
\end{aligned}
$$

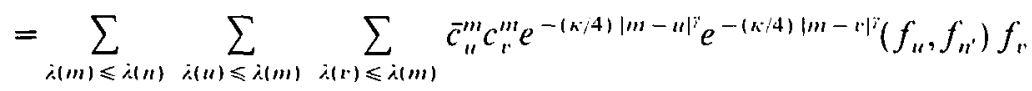

$$
\begin{aligned}
& =\sum_{\lambda(n) \leqslant \lambda(n)} \sum_{\lambda(u) \leqslant \lambda(n)} \sum_{\lambda(n) \geqslant \lambda(m) \geqslant \max (\lambda(u) . \lambda(r))} \bar{c}_{\|}^{m} c_{r}^{\prime \prime \prime} e^{-(\kappa / 4)|m-n|^{\prime}} \\
& \times e^{-(\kappa / 4)|m-1| \gamma}\left(f_{u}, f_{n^{\prime}}\right) f_{r}
\end{aligned}
$$

Therefore, if $\lambda\left(n^{\prime}\right)=\lambda(n)+1$, by $(3.3)$,

$$
g_{n^{\prime}}=\sum_{\lambda\left(r^{\prime}\right) \leqslant \lambda\left(n^{\prime}\right)} c_{r^{\prime}}^{n^{\prime}} e^{-\left(n^{\prime} / 4\right)\left|n^{\prime}-r\right|^{i}} f_{r}
$$


where $c_{n^{\prime}}^{n^{\prime}}=D_{n^{\prime}}^{-1 / 2}>1$ by (3.4) and for $\lambda(v)<\lambda\left(n^{\prime}\right)$,

$$
\begin{aligned}
& c_{n^{\prime}}^{\mu^{\prime}}=-D_{n^{\prime}}^{-1 / 2} \sum_{\lambda(u) \leqslant \lambda(n)} \sum_{\lambda(n) \geqslant \lambda(m) \geqslant \max (\lambda(u), \lambda(r))} \bar{c}_{\| \prime \prime}^{\prime \prime \prime} c_{n}^{\prime \prime} e^{-(\kappa / 4)|m-w|^{\prime}} \\
& \times e^{-(\kappa / 4)|m-r|^{\prime}} e^{(\kappa / 4)\left|n^{\prime}-r\right|^{\ddot{2}}}\left(f_{n}, f_{n^{\prime}}\right)
\end{aligned}
$$

Therefore

$$
\begin{aligned}
& \left|c_{r^{\prime \prime}}^{\prime \prime}\right| \leqslant 4 D_{n^{\prime}}^{-1 / 2} \sum_{\lambda(u) \leqslant \lambda(n) \quad \lambda(n) \geqslant \lambda(m) \geqslant \max (\lambda(u) \cdot \lambda(r))} \sum^{-(n / 4)|m-u|^{\prime}} \\
& x e^{-\left(w^{\prime} / 4\right)|m-r|^{\prime}} e^{(\kappa / 4) \mid n^{\prime}-r^{\prime \prime}}\left|\left(f_{t^{\prime}}, f_{n^{\circ}}\right)\right| \\
& \leqslant 4 D_{n^{\prime}}^{-1 / 2} C_{0}(\gamma) \sum_{\lambda(u) \leqslant \lambda(m)} e^{-(\kappa / 4)|n-r| \gamma} e^{(\kappa / 4)\left|n^{\prime}-r\right| \gamma}\left|\left(f_{u}, f_{n^{\prime}}\right)\right|
\end{aligned}
$$

by Lemma 3.3. We have, for $\left|u-n^{\prime}\right| \geqslant 1$,

$$
\begin{aligned}
\left|\left(f_{u,}, f_{n^{\prime}}\right)\right| & =e^{-\kappa\left|u-n^{\prime}\right|^{2}} \\
& =e^{-(\kappa / 4)\left|u-n^{\prime}\right|^{2}} e^{-(\kappa / 2)\left|u-n^{\prime}\right|^{2}} e^{-(\kappa / 4)\left|u-n^{\prime}\right|^{2}} \\
& \leqslant e^{-(\kappa / 4)\left|u-n^{\prime}\right|^{2}} e^{-\kappa / 2} e^{-\left(\kappa^{-} / 4\right)\left|u-n^{\prime}\right|^{i}}
\end{aligned}
$$

Also $\left|z_{1}\right|^{\gamma}+\left|z_{2}\right|^{\gamma}>\left|z_{1}+z_{2}\right|^{2}$; thus

$$
\left|c_{r}^{n^{\prime}}\right| \leqslant 4 D_{n^{\prime}}^{-1 / 2} C_{0}\left(\gamma^{\prime}\right) e^{-\kappa / 2} \sum_{\lambda(m) \leqslant \lambda(m)} e^{-(\kappa / 4)\left|n-n^{\prime}\right|^{2}} \leqslant 4 D_{n^{\prime}}^{-1 / 2} C_{n}\left(\gamma^{\prime}\right) K(\kappa / 4) e^{-\kappa / 2}
$$

Therefore if $\kappa$ is sufficiently large so that $8 C_{0}\left(\gamma^{\prime}\right) K(\kappa / 4) e^{-\kappa / 2}<1$, then $\left|c_{r}^{n^{\prime}}\right| \leqslant \frac{1}{2} D_{n^{\prime}}^{-1 / 2}$. But

$$
\left|\left(g_{m}, f_{n^{\prime}}\right)\right| \leqslant 2 \sum_{\lambda(u) \leqslant \lambda(m)} e^{-\left(\kappa^{\prime} / 4\right)|m-u|^{i}}\left|\left(f_{u}, f_{n^{\prime}}\right)\right| \leqslant 2 e^{-(\kappa / 4)\left|m-n^{\prime}\right| i} K(3 \kappa / 4)
$$

Thus

$$
\sum_{\lambda(m)<i\left(n^{\prime}\right)}\left|\left(g_{m}, f_{n^{\prime}}\right)\right|^{2} \leqslant 4 K(3 \kappa / 4)^{2} e^{-\kappa^{\prime} / 4} \sum_{m} e^{-(\kappa / 4)\left|m-n^{\prime}\right|} \leqslant e^{-\kappa / 8}<3 / 4
$$

if $k$ is large enough. Then $D_{n^{\prime}}>1 / 4$, so that $\left|c^{\prime \prime^{\prime}}\right| \leqslant 1$ for $\lambda(v)<\lambda\left(n^{\prime}\right)$. Using $(1-\varepsilon)^{-1 / 2}<1+\varepsilon$ for $\varepsilon<1 / 2$ and $D_{n^{\prime}}>1-e^{-\kappa / 8}$, we get also $c^{\prime \prime \prime} \leqslant 1+e^{-\kappa / 8}$. 
This last lemma allows us to obtain bounds on $\left|g_{n}(z)\right|$ and on the inner products $\left(g_{m}, f_{n}\right)$. We first obtain a bound on $\left|g_{n}(z)\right|$.

Corollary 3.5. For $k$ sufficiently large

$$
\left|g_{n}(z)\right| \leqslant e^{-\kappa ; 32} e^{-(\kappa / 12)|n-z|^{\prime}}+\sqrt{\frac{2 \kappa}{\pi}} e^{-\kappa^{*}|n-z|^{2}}
$$

Proof. We have

$$
\left|g_{n}(z)-f_{n}(z)\right| \leqslant\left(c_{n}^{n}-1\right)\left|f_{n}(z)\right|+\sum_{\lambda(m)<i(n)}\left|c_{m}^{\prime \prime}\right| e^{-(\kappa / 4)|n-m|^{\mid}}\left|f_{m}(z)\right|
$$

Now

$$
\begin{aligned}
\left|f_{n}(z)\right| & =\sqrt{\frac{2 \kappa}{\pi}} e^{-\kappa|n-z|^{2}} \\
& =\sqrt{\frac{2 \kappa}{\pi}} e^{-\left.(\kappa ; 12)|n-=|\right|^{*}} e^{(\kappa / 12)|n-z|^{\prime}} e^{-\kappa \cdot 1 n-\left.z\right|^{2}} \\
& \leqslant \sqrt{\frac{2 \kappa}{\pi}} e^{-(\kappa / 12)|n-z|^{*}} e^{\kappa / 12}
\end{aligned}
$$

since $|z|^{2}>-\delta+\delta|z|^{y}$ for $0<\delta<1$. Therefore

$$
\left(c_{n}^{n}-1\right)\left|f_{n}(z)\right| \leqslant e^{-\kappa / 24} \sqrt{\frac{2 \kappa}{\pi}} e^{-(\kappa / 12)|n-z|}
$$

In the sum (3.16), $|n-m| \geqslant 1$, so that

$$
\begin{aligned}
\frac{\kappa}{4} \mid n- & \left.m\right|^{y}+\kappa|m-z|^{2} \\
& \geqslant \frac{\kappa}{6}+\frac{\kappa}{12}|n-m|^{\prime}+\kappa|m-z|^{2} \\
& \geqslant \frac{\kappa}{6}+\frac{\kappa}{12}|n-z|^{\gamma}-\frac{\kappa}{12}|m-z|^{\gamma}+\frac{\kappa}{2}|m-z|^{2}+\frac{\kappa}{2}|m-z|^{2} \\
& \geqslant \frac{\kappa}{12}+\frac{\kappa}{12}|n-z|^{\prime}+\frac{\kappa}{2}|m-z|^{2}
\end{aligned}
$$


Therefore for $\kappa$ sufficiently large

$$
\sum_{\lambda(m)<\lambda(n)}\left|c_{m}^{\prime \prime}\right| e^{-(\kappa / 4)|n-m|^{i}}\left|f_{m}(z)\right| \leqslant e^{-\kappa / 16} e^{-(\kappa / 12)|n-z|^{i}}
$$

Thus

$$
\left|g_{n}(z)-f_{n}(z)\right| \leqslant e^{-\kappa / 32} e^{-(\kappa / 12)|n-z|^{\prime}}
$$

and therefore

$$
\left|g_{n}(z)\right| \leqslant e^{-\kappa / 32} e^{-(\kappa / 12)|n-z| r}+\sqrt{\frac{2 \kappa}{\pi}} e^{-\kappa|n-z|^{2}}
$$

The bound on $\left|\left(g_{m}, f_{n}\right)\right|$ now follows very easily.

Corollary 3.6. For $\kappa$ sufficiently large

$$
\left|\left(g_{m}, f_{n}\right)\right| \leqslant 4 e^{-(\kappa / 4)|m-n|^{*}}
$$

Proof. By Lemma 3.4

$$
\begin{aligned}
\left|\left(g_{m}, f_{n}\right)\right| & \leqslant 2 \sum_{\lambda(u) \leqslant \lambda(m)} e^{-(\kappa / 4)|m-u|^{i}}\left|\left(f_{u}, f_{n}\right)\right| \\
& \leqslant 2 \sum_{\lambda(u) \leqslant \lambda(m)} e^{-(\kappa / 4)|m-u|^{i}} e^{-\kappa|n-u|^{2}} \leqslant 2 e^{-(\kappa / 4)|m-n|^{\prime} K(3 \kappa / 4)}
\end{aligned}
$$

We first establish the bounds at the beginning of Theorem 3.2.

Lemma 3.7. For $k$ large enough there exists $A>0$ independent of $\kappa$ such that

$$
\begin{gathered}
\left|\left\langle n\left|\hat{H}_{A}\right| m\right\rangle\right| \leqslant A e^{-(\kappa / 8)|n-m| i} \\
\left\|\hat{H}_{A}\right\| \leqslant A
\end{gathered}
$$

and

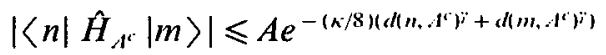

for all $\Lambda \subset \mathbb{Z}[i]$. 
Proof. We have

$$
\begin{aligned}
\left|\left\langle n\left|\hat{H}_{A}\right| m\right\rangle\right| & =\left|\sum_{u \in A} \omega_{u}\left(g_{u}, f_{u}\right)\left(f_{u}, g_{m}\right)\right| \\
& \leqslant M^{\prime} \sum_{u \in \mathbb{Z}[i]} e^{-(\kappa / 4) \mid u-\|^{i}} e^{-(\kappa / 4)|u-m|^{i}} \\
& \leqslant M^{\prime} C_{0}(\gamma) e^{-(\kappa / 4)|n-m|^{*}}
\end{aligned}
$$

where $M^{\prime}=16 M$. Let $S$ be the operator on $\mathscr{M}_{1}$ with $\langle n|S| m\rangle=$ $e^{-\left.(\kappa / 4)|n-m|\right|^{\prime}}$. Then $\left\|\hat{H}_{, d}\right\| \leqslant M^{\prime} C_{0}(\gamma)\|S\|$. Finally,

$$
\begin{aligned}
& \left|\left\langle n\left|\hat{H}_{A^{\prime}}\right| m\right\rangle\right|=\left|\sum_{u \in A^{\prime}} \omega_{u}\left(g_{n}, f_{u}\right)\left(f_{u}, g_{m}\right)\right| \\
& \leqslant M^{\prime} \sum_{u \in A^{\prime \prime}} e^{-(\kappa / 4)|u-\mu|^{i}} e^{-(\kappa / 4)|u-m|^{i}}
\end{aligned}
$$

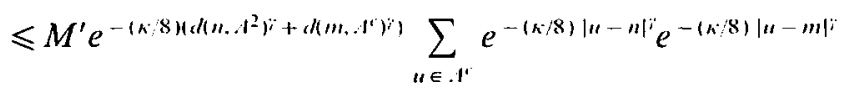

$$
\begin{aligned}
& \leqslant M^{\prime} C_{0}(\gamma) e^{-(k / k)\left(d / n \cdot d^{\prime}\right)+d\left(m \cdot A^{\prime}\right)}
\end{aligned}
$$

Let $\mathscr{E}_{1}^{*}$ be the set of functions on $\mathbb{C}$ of the form

$$
\phi(z)=\sum_{n \in \mathbb{Z}[i]} a_{n} g_{n}(z)
$$

where $\left|a_{n}\right|(1+|n|)^{-1}$ is bounded for some $t \in \mathbb{N}$. Then $\mathscr{E}_{1}^{*}$ is the dual of the nuclear space $\mathscr{E}_{1}$ consisting of functions on $\mathbb{C}$ of the same form with $\left|a_{n}\right| \cdot|n|^{\prime}$ bounded for all $t \in \mathbb{N}$, equipped with the seminorms $\|\cdot\|_{l}, t \in \mathbb{N}$, defined by

$$
\|\phi\|_{i}^{2}=\sum_{i^{\prime}=0}^{\prime} \sum_{n \in \mathbb{Z}[i]}\left|a_{n}\right|^{2}|n|^{2 t^{\prime}}
$$

This is a nuclear space since $\sum_{n \in \mathbb{Z}[i]}\left(\left\|g_{n}\right\| /\left\|g_{l}\right\|_{t^{\prime}}\right)$ converges for $t^{\prime}>t+2$ (ref. 17, p. 62). Lemma 3.7 gives the bound

$$
\left.\mid\left\langle n\left|H_{1}\right| m\right\rangle\right\} \mid \leqslant A e^{-(\kappa / 8)|n-m|^{i}}
$$

This implies that $H_{1}$ maps $\mathscr{E}_{1}$ continuously onto itself, which allows us to define $H_{1}$ on $\mathscr{E}_{1}^{*}$ by duality as the adjoint map. The generalized eigenfunctions of $H_{1}$ are then in $\mathscr{E}_{1}^{*}$ and therefore polynomially bounded, that is, $\left|a_{n}\right|<C(1+|n|)^{\prime(4)}$ 
The next lemma will be used in proving that (P1) and (RA) of (P2) in Theorem 3.2 are satisfied.

Lemma 3.8. If $x_{1}, \ldots, x_{n}$ are i.i.d. random variables with density $\rho$ bounded by $\rho_{0}$, then $\tilde{\rho}$, the density of $\sum_{k=1}^{n} \alpha_{k} x_{k}, \alpha_{k}>0, k=1, \ldots, n$, is bounded by $\rho_{0} / \max \alpha_{k}$.

Proof. We have

$$
\begin{aligned}
\tilde{\rho}\left(y_{1}\right)= & \frac{1}{\alpha_{1}} \int \rho\left(\frac{y_{1}-y_{2}}{\alpha_{1}}\right) \rho\left(\frac{y_{2}-y_{3}}{\alpha_{2}}\right) \cdots \rho\left(\frac{y_{n-1}-y_{n}}{\alpha_{n-1}}\right) \\
& \times \rho\left(\frac{y_{n}}{\alpha_{n}}\right) \frac{d y_{2}}{\alpha_{2}} \ldots \frac{d y_{n}}{\alpha_{n}} \\
\leqslant & \frac{\rho_{0}}{\alpha_{1}} \int \rho\left(\frac{y_{2}-y_{3}}{\alpha_{2}}\right) \rho\left(\frac{y_{3}-y_{4}}{\alpha_{3}}\right) \cdots \rho\left(\frac{y_{n-1}-y_{n}}{\alpha_{n-1}}\right) \\
& \times \rho\left(\frac{y_{n}}{\alpha_{n}}\right) \frac{d y_{2}}{\alpha_{2}} \ldots \frac{d y_{n}}{\alpha_{n}} \\
= & \frac{\rho_{0}}{\alpha_{1}} \int \rho\left(z_{2}\right) \rho\left(z_{3}\right) \cdots \rho\left(z_{n}\right) d z_{2} \cdots d z_{n}=\frac{\rho_{0}}{\alpha_{1}}
\end{aligned}
$$

We can write $H_{A}$ in the form

$$
H_{A}=\sum_{m, m^{\prime} \in A} x_{m, m^{\prime}} g_{m} \otimes \bar{g}_{m^{\prime}}
$$

where $x_{m m^{\prime}}=\sum_{n \in A} \omega_{n}\left(g_{m}, f_{n}\right)\left(f_{n}, g_{m}\right)$. Since

$$
x_{m m n}=\sum_{n \in d} \omega_{n}\left|\left(g_{m}, f_{n}\right)\right|^{2}
$$

by the preceding lemma $x_{m, ! m}$ has a density bounded by $\rho_{0} /\left|\left(g_{m}, f_{m}\right)\right|^{2}$, where $\rho_{0}$ is the upper bound for the density of the $\omega_{n}$. Now, using Lemma 3.4, we have

$$
\left|\left(f_{m}, g_{m}\right)\right|^{2}=D_{m}=\left(c_{m}^{\prime \prime \prime}\right)^{-2}>\left(1+e^{-\kappa / 8}\right)^{-2}>1 / 2
$$

for $\kappa$ sufficiently large. Thus $x_{m, m}$ has a density bounded by $2 \rho_{0}$. For Borel subsets $B$ of $\mathbb{R}$ let $\sigma_{m}^{d}(B)=\left\langle m\left|E_{A}(B)\right| m\right\rangle$, were $E_{d}(B)$ are the spectral projections of $H_{A}$. Then by Lemma VIII.1.8 in ref. 14 (in ref. 14 the lemma 
is proven for real matrices, but one can easily check that it extends to the present situation where only the diagonal elements are real),

$$
\mathbb{E}_{x_{m m}} \sigma_{m}^{A}(B)<2 \rho_{0} \int_{B} d x
$$

and therefore

$$
\mathbb{E} \sigma_{m}^{\prime \prime}(B)<2 \rho_{0} \int_{B} d x
$$

As in Proposition VIII.4.11 of ref. 14, it then follows that for all $E \in \mathbb{R}$ and $\varepsilon>0$,

$$
\mathbb{P}\left(d\left(E, \sigma\left(H_{A}\right)\right)<\varepsilon\right)<4 \rho_{0} \varepsilon|\Lambda|
$$

The next lemma will be used in proving that (RB) of (P2) in Theorem 3.2 is satisfied. Let

$$
\widetilde{H}_{A}=\sum_{n \in A} \omega_{n} g_{n} \otimes \bar{g}_{n}
$$

and $\tilde{G}_{d}(E)=\left(E-\tilde{H}_{d}\right)^{-1}$. If $d\left(E,\left\{\omega_{n}: n \in A\right\}\right)>\varepsilon$, then we have

$$
\left|\left\langle n\left|\widetilde{G}_{d}(E)\right| m\right\rangle\right| \leqslant \frac{1}{\varepsilon} \delta_{m . n}
$$

Lemma 3.9. Given $\varepsilon>0$, if $d\left(E,\left\{\omega_{n}: n \in A\right\}\right)>\varepsilon$ and if $k$ is large enough so that $e^{-\kappa / 32}<\varepsilon / 2$, then

$$
\left|\left\langle n\left|G_{A}(E)\right| m\right\rangle\right| \leqslant \varepsilon^{-1}\left(\delta_{n, m}+e^{-(\kappa / 8)|m-m|^{i}}\right)
$$

Proof. Let $\delta H_{A}=H_{A}-\widetilde{H}_{A}$. If $n \neq m$, then $\left|\left\langle n\left|\tilde{H}_{A}\right| m\right\rangle\right|=0$; therefore by Corollary 3.6 and Lemma 3.3, for $\kappa$ large enough,

$$
\begin{aligned}
\left|\left\langle n\left|\delta H_{A}\right| m\right\rangle\right| & =\left|\sum_{u \in A} \omega_{u}\left(g_{n}, f_{u}\right)\left(f_{u}, g_{m}\right)\right| \\
& \leqslant M^{\prime} \sum_{u \in \mathbb{Z}[i]} e^{-(\kappa / 4)|u-n|^{i}} e^{-(\kappa / 4)|u-m|^{i}} \\
& \leqslant M^{\prime} C_{0}(\gamma) e^{-\kappa / 8} e^{-(\kappa / 8)|m-m|^{r}}
\end{aligned}
$$


On the other hand,

$$
\begin{aligned}
\left|\left\langle n\left|\delta H_{A}\right| n\right\rangle\right| & =\left.\left|\sum_{u \in A} \omega_{u}\right|\left(f_{u}, g_{n}\right)\right|^{2}-\omega_{n} \mid \\
& \leqslant M \sum_{u \neq n}\left|\left(f_{u}, g_{n}\right)\right|^{2}+M\left(1-\left|\left(f_{n}, g_{n}\right)\right|^{2}\right) \\
& =M \sum_{u \neq n}\left|\left(f_{u}, g_{n}\right)\right|^{2}+M \sum_{\lambda(u)<i(n)}\left|\left(f_{u}, g_{u}\right)\right|^{2} \\
& \leqslant M \sum_{u \neq n}\left|\left(f_{u}, g_{n}\right)\right|^{2}+M \sum_{u \neq n}\left|\left(f_{n}, g_{u}\right)\right|^{2} \\
& \leqslant 2 M^{\prime} \sum_{u \neq n} e^{-(\kappa / 2)|u-n|^{i}} \\
& \leqslant 2 M^{\prime} e^{-\kappa / 8} \sum_{u \in \mathbb{Z}[i]} e^{-(3, / 8)|u|^{i}}
\end{aligned}
$$

Therefore for $\kappa$ large enough

$$
\left|\left\langle n\left|\delta H_{,}\right| m\right\rangle\right| \leqslant e^{-\kappa / 16} e^{-(\kappa / 8)|n-m| i}
$$

Thus

$$
\left|\left\langle n\left|\widetilde{G}_{.1}(E) \delta H_{A}\right| m\right\rangle\right|=\left|E-\omega_{n}\right|^{-1}\left|\left\langle n\left|\delta H_{A}\right| m\right\rangle\right| \leqslant \varepsilon^{-1} e^{-\kappa / 16} e^{-(\kappa / 8)|n-m|^{\prime}}
$$

By using Lemma 3.3 we can then deduce that

$$
\begin{aligned}
\left|\left\langle n\left|\left(\widetilde{G}_{A}(E) \delta H_{A}\right)^{k}\right| m\right\rangle\right| & \leqslant\left(C_{0}(\gamma)\right)^{k-1} \varepsilon^{-k} e^{-k \kappa / 16} e^{-(\kappa / 8)|n-m|^{i}} \\
& \leqslant \varepsilon^{-k} e^{-k \kappa / 32} e^{-\langle\kappa / 8)|n-m| \eta} \leqslant \frac{1}{2^{k}} e^{-(\kappa / 8)|n-m|^{i}}
\end{aligned}
$$

From the resolvent identity we get

$$
G_{A}(E)=\tilde{G}_{.1}(E)+\tilde{G}_{A}(E) \delta H_{A} G_{A}(E)=\sum_{k=0}^{\infty}\left(\widetilde{G}_{A}(E) \delta H_{A}\right)^{k} \widetilde{G}_{A}(E)
$$

if the series converges. Let $T$ be the operator with $\langle n|T| m\rangle=$ $\exp \left[-(\kappa / 8)|n-m|^{\gamma}\right]$. Then

$$
\left\|\left(\tilde{G}_{A}(E) \delta H_{A}\right)^{k}\right\| \leqslant \frac{1}{2^{k}}\|T\|
$$


and therefore the series converges. Thus

$$
\left|\left\langle n\left|G_{A}(E)\right| m\right\rangle\right| \leqslant \varepsilon^{-1}\left(\delta_{n, m}+e^{-(\kappa / 8)|n-m|^{*}}\right)
$$

We are now ready to show that the conditions of Theorem 3.2 are satisfied. We have proved that there is $\kappa_{0}>0$ such that for all $\kappa>\kappa_{0}$ and for all $A \subset \mathbb{Z}[i]$, the following are satisfied: (1)

$$
\begin{aligned}
\left|\left\langle n\left|\hat{H}_{A}\right| m\right\rangle\right| & \leqslant A e^{-\left(\hat{k}_{0} / 8\right)|n-m|^{*}} \\
\left\|\hat{H}_{A}\right\| & \leqslant A
\end{aligned}
$$

and

$$
\left|\left\langle n\left|\hat{H}_{A^{c}}\right| m\right\rangle\right| \leqslant A e^{-\left(\kappa_{0} / 8\right)\left(d\left(n \cdot A^{c}\right)^{i}+d\left(m \cdot A^{n} \eta^{n}\right)\right.}
$$

(2) For all $E \in \mathbb{R}$ and $\varepsilon>0$,

$$
\mathbb{P}\left(d\left(E, \sigma\left(H_{.1}\right)\right)<\varepsilon\right)<4 \rho_{0} \varepsilon|\Lambda|
$$

(3) If $d\left(E,\left\{\omega_{n}: n \in A\right\}\right)>\varepsilon$ and $e^{-\kappa / 32}<\varepsilon / 2$, then

$$
\left|\left\langle n\left|G_{A}(E)\right| m\right\rangle\right| \leqslant \varepsilon^{-1}\left(\delta_{n, m}+e^{-\left(\text {kili }_{1} / 8\right)|n-m|^{\mid}}\right)
$$

From (2) we have that, for all $E \in \mathbb{R}$ and for all $L>0$,

$$
\mathbb{P}\left(d\left(E, \sigma\left(H_{A_{L}(n)}\right)\right)<\varepsilon^{-L^{H}}\right)<4 \rho_{0} e^{-L^{H}} L^{2}
$$

so that if we take $L_{0}$ sufficiently large, for all $L>L_{0},(\mathrm{P} 1)$ is satisfied for any $q>0$. Also the probability that (RA) of (P2) is satisfied is greater than $1-\frac{1}{2} L^{-q}$.

Now let $L_{1}$ be as in Theorem 3.2 and choose $L_{0}>L_{1}$ such that

$$
L_{0}^{6} e^{\left.-\left(\kappa_{i}\right) / 8\right)\left(L_{i 1}-L_{i j}^{i}\right)^{\prime}}<e^{-\left(\kappa_{i j} / 16\right) L_{i}^{i}}
$$

and

$$
\left(1-\frac{2 \rho_{0}}{L_{0}^{6}}\right)^{L_{0}^{2}}-\frac{1}{2 L_{0}^{4}}>1-\frac{1}{L_{0}^{3}}
$$

Then from (3), by putting $\varepsilon=1 / L_{0}^{6}$, we see that if $\kappa>\kappa_{1} \equiv$ $\max \left(\kappa_{0}, 32 \ln \left(2 L_{0}^{6}\right)\right)$, then $(\mathrm{RB})$ of $(\mathrm{P} 2)$ is satisfied with probability greater than $1-1 / L_{0}^{3}+1 /\left(2 L_{0}^{4}\right)$. Thus the probability that (RA) and (RB) are satisfied is greater than $1-1 / L_{0}^{3}$; that is, (P2) holds with $p=3$. 


\section{THE PROOF OF THEOREM 3.2}

This short section deals with the proof of Theorem 3.2. Our proof is based on the paper of von Dreifus and Klein ${ }^{(13)}$ as adapted, in our paper, ${ }^{(4)}$ to infinite-range operators, that is, operators that couple points within a region to points outside the region. Here we give only the modifications that have to be made to the method of ref. 4 to fit the present situation; this section should be read in conjunction with Sections 4,6 , and 7 in that paper. Some simplification occurs because in this paper we require less than exponential decay. Theorem 3.2 is the analogue of Theorem 4.1 in ref. 4. As before, this theorem can be split up into two parts: one in which condition (P2) is iterated to pairs of larger and larger blocks (Theorem 4.9) and one in which the iterated condition is shown to imply the required decay of the eigenfunctions (Theorem 4.1). The following theorem is the analogue of Theorem 4.2 in ref. 4 . However, the proof is much simpler than that of the latter and therefore we give it.

Theorem 4.1. Let $0<\gamma^{\prime}<\gamma_{0}$ and let $I \subset \mathbb{R}$ be an interval. Let $1<\alpha<\min \left(2 s, \gamma_{0} s / \beta, \gamma_{0} / \gamma^{\prime}\right), \quad r>0, \quad$ and $L_{0}>1$. Define $L_{k+1} \equiv L_{k}^{\alpha}$, $k=0,1,2, \ldots$. Suppose that for all $k=0,1,2, \ldots$ and all $n, m \in \mathbb{Z}[i]$ with $|n-m|_{x} \geqslant L_{k}+1$,

$\mathbb{P}\left\{\exists E \in I\right.$ such that $\Lambda_{L_{k}}(n)$ and $\Lambda_{L_{k}}(m)$ are both $(r, E)$-singular $\}<L^{-2 p}$

Then, with probability one, the generalized eigenfunctions of $H_{1}$ corresponding to generalized eigenvalues in $I$ satisfy

$$
\lim _{n \rightarrow \infty} \frac{\ln \left|\left(g_{n}, \psi\right)\right|}{|n|^{r^{\prime}}}=-\infty
$$

Note that the last inequality together with Corollary 3.5 implies that

$$
\lim _{z \rightarrow \infty} \frac{\ln |\psi(z)|}{|z|^{\gamma^{\prime}}}=-\infty
$$

We need the following two lemmas, the analogues of Lemma 6.3 in ref. 4 , which we state without proof. We find it more convenient here to give estimates for the two operators which occur in the proof of Theorem 4.1 than a general estimate as in ref. 4. Throughout the rest of this section $R$ is as in (3.9).

Lemma 4.2. Let $\psi$ be a generalized eigenfunction of $H_{1}$ with eigenvalue $E$ and therefore polynomially bounded, that is, there exist $K>0$ and 
$t \in \mathbb{N}$ such that $\left|\left(g_{n}, \psi\right)\right| \leqslant K(1+|n|)^{\prime}$. If $A_{L}\left(n_{0}\right)$ is $(r, E)$-regular for some $r>0$, then there exists a constant $A_{1}$ such that

$$
\begin{aligned}
& \left|\left(g_{m, 1}, G_{A_{L}(m, 1)}(E) H_{1} P_{A_{L\left(m_{1}\right)}} \psi\right)\right| \\
& \leqslant A_{1}\left\{\left(L+\left|n_{0}\right|\right)^{\prime} e^{-R L / 2}+e^{-r L n} \sum_{n \in A_{2 L}\left(\mu_{n}\right)}\left|\left(g_{n}, \psi\right)\right|\right\}
\end{aligned}
$$

for $L$ large enough.

Lemma 4.3. Let $\psi$ be a generalized eigenfunction of $H_{1}$ with eigenvalue $E$. If $\Lambda_{L}\left(n_{0}\right)$ is $(r, E)$-regular for some $r>0$, then there exist $A_{2}>0$ and $Q_{2}$ such that for $L>Q_{2}$

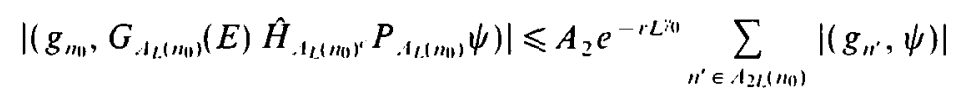

We now give the proof of Theorem 4.1.

Proof of Theorem 4.1. Define $A_{k+1}\left(n_{0}\right) \equiv A_{2 L_{k+1}}\left(n_{0}\right) \backslash A_{2 L_{k}}\left(n_{0}\right)$ and let $\mathscr{E}_{k}\left(n_{0}\right)$ be the set of $\omega \in \Omega$ for which there exists $n \in A_{k+1}\left(n_{0}\right)$ such that $A_{L_{k}}\left(n_{0}\right)$ and $A_{L_{k}}(n)$ are both $(r, E)$-singular for some $E \in I$. By the Borel-Cantelli Lemma we may assume that for all $n_{0} \in \mathbb{Z}[i]$, the set $\left\{k: \omega \in \mathscr{E}_{k}\left(n_{0}\right)\right\}$ has a finite number of elements. Now let $\psi$ be a generalized eigenfunction of $H_{1}$ with eigenvalue $E$ and choose $n_{0} \in \mathbb{Z}[i]$ such that $\left|\left(g_{n_{1}}, \psi\right)\right| \neq 0$. By the resolvent identity,

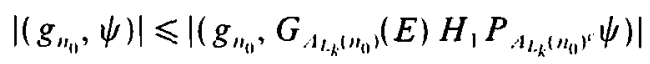

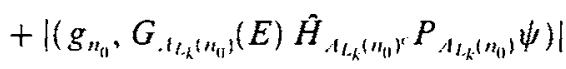

Suppose that $A_{L_{k}}\left(n_{0}\right)$ is $(r, E)$-regular. Then, using Lemmas 4.2 and 4.3 , the last inequality implies that

$$
\begin{aligned}
\left|\left(g_{n_{1 n^{\prime}}}, \psi\right)\right| \leqslant & A_{1}\left(L_{k}+\left|n_{0}\right|\right)^{\prime} e^{-(1 / 2) R L_{k}^{\prime \prime}} \\
& +\left(A_{1}+A_{2}\right) e^{-r L_{k}^{\prime \prime \prime}} \sum_{n^{\prime} \in A_{2 L_{k}\left(n_{0}\right)}}\left|\left(g_{n^{\prime}}, \psi\right)\right|
\end{aligned}
$$

If this were to hold for infinitely many $k$ 's, we could take the limit $k \rightarrow \infty$ and find that $\left|\left(g_{n_{0}}, \psi\right)\right|=0$, which is a contradiction. We conclude that there exists $k_{1}$ such that for all $k \geqslant k_{1}, \Lambda_{L_{k}}\left(n_{0}\right)$ is $(r, E)$-singular. This implies that if $\omega \in \Omega_{0}=\bigcup_{k=1}^{\infty} \mathscr{E}_{k}\left(n_{0}\right)$, then there exists $k_{2} \geqslant k_{1}$ such that for 
$k \geqslant k_{2}, A_{L_{k}}(n)$ is $(r, E)$-regular for all $n \in A_{k+1}\left(n_{0}\right)$. The above inequality with $n_{0}$ replaced by $n$ then yields

$$
\begin{aligned}
\left|\left(g_{n}, \psi\right)\right| \leqslant & A_{1}\left(L_{k}+|n|\right)^{\prime} e^{-(1 / 2) R L_{k}^{\prime}} \\
& +\left(A_{1}+A_{2}\right) e^{-r L_{k}^{j_{k}^{\prime \prime}}} \sum_{n^{\prime} \in A_{2 L_{k}(l n)}\left|\left(g_{n^{\prime}}, \psi\right)\right|} \\
\leqslant & C e^{-\left.r^{\prime}|n|^{\prime \prime \prime}\right|^{2}}
\end{aligned}
$$

where $r^{\prime}<r$, since $\left|n-n_{0}\right| \leqslant L_{k+1}=L_{k}^{\alpha}$.

The next three lemas are the analogues of Lemma 7.4 of ref. 4 . The first two are equivalent to proving the inequalities 7.27 and 7.32 in Lemma 7.4 and are sufficiently close to the these inequalities that they do not require a proof here; the third one is significantly different and warrants a proof. The difference is mainly due to the fact that we do not require exponential decay. As a result the parameter $r$ in the regularity condition can be fixed for all scales. We first need to define the following two conditions.

Let $I \subset \mathbb{R}$ be a fixed interval.

$R\left(L, r^{\prime}\right):$ For all $n, m \in \mathbb{Z}[i]$ with $|n-m|_{x} \geqslant L+1$,

$\mathbb{P}\left\{\exists E \in I: \Lambda_{L}(n)\right.$ and $\Lambda_{L}(m)$ are both $\left(r^{\prime}, E\right)$-singular $\}<L^{-2 p}$

$K\left(L_{0}\right):$ For all $L \geqslant L_{0}$, all $E \in \mathbb{R}$ with $d(E, I) \leqslant \frac{1}{2} \exp \left(-L^{\beta}\right)$, and all $n \in \mathbb{Z}[i]$,

$$
\mathbb{P}\left\{d\left(E, \sigma\left(H_{A_{L}(n)}\right)\right)<e^{-L^{\beta}}\right\}<L^{-\imath}
$$

Lemma 4.4. Let $l>1$ and $L=l^{x}$. Assume that $\Lambda_{l}(n) \subset \Lambda_{L}\left(n_{0}\right)$ is $(r, E)$-regular. Then, if $l$ is large enough, there exist constants $C_{1}$ and $\tilde{A}_{1}$ such that for $m \in \partial \Lambda_{L}\left(n_{0}\right)$,

$$
\begin{aligned}
\mid\left\langle n\left|G_{A_{L}\left(n_{0}\right)}(E)\right| m\right\rangle \leqslant & C_{1} \exp \left[-\frac{1}{4} R l^{\psi s}\right]+\tilde{A}_{1} l^{s+1} e^{-r l_{10}} \\
& \times\left\{e^{\alpha \beta \beta} \wedge \sup _{n^{\prime} \in A_{i}(n)}\left|\left\langle n^{\prime}\left|G_{A_{L}\left(n_{1}\right)}(E)\right| m\right\rangle\right|\right\}
\end{aligned}
$$

where $\hat{l}=l+l^{s}+1$.

Lemma 4.5. Let $l^{\prime} \geqslant l$ and $L=l^{x}$. Suppose that $A_{l^{\prime}}\left(n_{1}\right) \subset \Lambda_{L}\left(n_{0}\right)$ and that $\Lambda_{1},\left(n_{1}\right)$ and $\Lambda_{L}\left(n_{0}\right)$ satisfy the regularity condition (RA). Then 
there exist constants $C_{2}$ and $\widetilde{A}_{2}$ such that for $l$ large enough, $n \in \Lambda_{l^{\prime}}\left(n_{1}\right)$, and $m \in \partial \Lambda_{L}\left(n_{0}\right)$,

$$
\begin{aligned}
& \left|\left\langle n\left|G_{t_{t,}\left(n_{n, 1}\right)}(E)\right| m\right\rangle\right| \\
& \leqslant C_{2} e^{-(R / 4) / r^{\prime s}}+\tilde{A}_{2} l^{\prime} e^{I^{\prime} / s} \sup _{n^{\prime} \in A_{i}\left(m_{1}\right) A_{i} \cdot\left(m_{1}\right)}\left|\left\langle n^{\prime}\left|G_{A_{L}\left(n_{0}\right)}(E)\right| m\right\rangle\right|
\end{aligned}
$$

where $\tilde{l}^{\prime}=l^{\prime}-l^{s}$.

Lemma 4.6. Let $J \in \mathbb{N}$ be given and $n_{0} \in \mathbb{Z}[i]$. There exists $Q^{\prime} \geqslant 1$ such that if $l \geqslant Q^{\prime}$ and $L=l^{x}$, then (i)-(iii) below imply that $A_{L}\left(n_{0}\right)$ is $(r, E)$-regular:

(i) $\Lambda_{L}\left(n_{0}\right)$ satisfies (RA).

(ii) $\Lambda_{r^{\prime}}\left(n^{\prime}\right)$ satisfies (RA) for all

$$
l^{\prime} \in\{l, l+\hat{l}+1, \ldots, J(l+\hat{l}+1)\} \equiv \mathscr{T}_{l}
$$

and all $n^{\prime} \in \Lambda_{L}\left(n_{0}\right)$ such that $\Lambda_{r}\left(n^{\prime}\right) \subset \Lambda_{L}\left(n_{0}\right)$.

(iii) There are at most $J$ squares $\Lambda_{I}\left(n_{i}\right) \subset \Lambda_{\tilde{L}}\left(n_{0}\right), i=1, \ldots, D \leqslant J$, such that $d\left(n_{i}, n_{j}\right) \geqslant l+1$ for $i \neq j$ which are $(r, E)$-singular.

Proof. Choose a maximal set of singular squares $A_{1}\left(n_{i}\right), i=1, \ldots$, $D \leqslant J$, in $A_{\tilde{L}}\left(n_{0}\right)$ as in (iii). Then if $\Lambda_{l}(n) \subset \Lambda_{\tilde{L}}\left(n_{0}\right)$ and $d\left(n, n_{i}\right) \geqslant l+1$ for all $i, A_{l}(n)$ is regular. There exist squares $A_{l_{i}} \subset A_{\tilde{L}}\left(n_{0}\right)$ with $l_{j} \in \mathscr{T}_{l}, j=1, \ldots$, $t \leqslant D$, such that $A_{t_{j}} \cap A_{l_{i}}=\varnothing$ for $i \neq j, \sum_{i=1}^{\prime} l_{i} \leqslant J(l+\hat{l}+1)$, and

$$
\bigcup_{i=1}^{r} A_{1+i+1}\left(n_{i}\right) \subset \bigcup_{i=1}^{i} A_{l}
$$

If $n \notin \Lambda_{\bar{l}_{i}}$ for any $j=1, \ldots, t$ and $\Lambda_{l}(n) \subset \Lambda_{\tilde{L}}\left(n_{0}\right)$, then $d\left(n, n_{i}\right) \geqslant l+1$ because $d\left(n, A_{l}^{c}\right) \leqslant \frac{1}{2} l^{*}$ and $d\left(n_{i}, A_{l}^{c}\right) \geqslant \frac{1}{2}(l+\hat{l}+1)$, which implies that $\left|n-n_{j}\right| \geqslant$ $\frac{1}{2}(l+\hat{l}+1)-\frac{1}{2} l^{*}=l+1$. Therefore $A_{l}(n)$ is regular. By Lemma 4.4 , if $\Lambda_{l}(n)$ is regular, then (4.8) is satisfied. On the other hand, if $\Lambda_{i}(n)$ is singular but $\Lambda_{f}(n) \subset \Lambda_{L}\left(n_{0}\right)$, then $\left|n-n_{i}\right| \leqslant l+1$. This implies that $n \in \Lambda_{1+i+1}\left(n_{i}\right)$ for some $i$ and therefore $n \in A_{i}$ for some $j$. Thus by Lemma 4.5,

$$
\begin{aligned}
& \left|\left\langle n\left|G_{A_{1},\left(n_{0}\right)}(E)\right| m\right\rangle\right| \\
& \leqslant C_{2} e^{-(R / 4) / j^{\prime s}}+\tilde{A}_{2} l_{j} e^{f_{j}^{\prime \prime}} \sup _{n^{\prime} \in A i_{j} \backslash M_{i j}}\left|\left\langle n^{\prime}\left|G_{A_{L}\left(n_{0}\right)}(E)\right| m\right\rangle\right|
\end{aligned}
$$


Since $n^{\prime} \notin \Lambda_{i_{j}}, \Lambda_{l}\left(n^{\prime}\right)$ is regular provided $\Lambda_{l}\left(n^{\prime}\right) \subset \Lambda_{L}\left(n_{0}\right)$. We can therefore use $(4.8)$ to write

$$
\begin{aligned}
& \left|\left\langle n\left|G_{A_{1},\left(n_{n}\right)}(E)\right| m\right\rangle\right| \leqslant C_{2} e^{-(R / 4) / m}+\tilde{A}_{2} l_{j} e^{l_{j}}\left\{C_{1} e^{-(R / 4) / m}\right.
\end{aligned}
$$

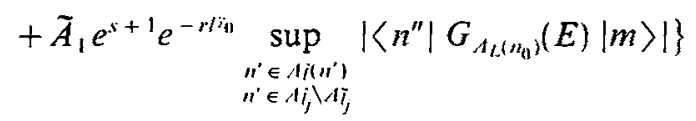

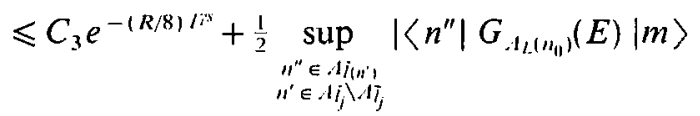

for $l$ large enough, depending on $J$. Note that we have used $l_{j} \leqslant 2 J l, \gamma_{0}>\beta$. We now want to iterate (4.8) and (4.11) starting at $n=n_{0}$ : This can be done until we reach $\partial \Lambda_{L}\left(n_{0}\right)$. Suppose we can perform $N$ iterations. Then we can write

$$
\begin{aligned}
\left|\left\langle n_{0}\left|G_{\lambda_{L}\left(n_{0}\right)}(E)\right| m\right\rangle\right| & \\
\leqslant & \bar{C}^{-(R / 4) / ; s}\left\{1+Z\left(n_{0}\right) \sum_{k=0}^{N-2} Z\left(n_{1}\right) \cdots Z\left(n_{k}\right)\right\} \\
& +Z\left(n_{0}\right) \cdots Z\left(n_{N-1}\right) e^{\alpha / / i}
\end{aligned}
$$

where

$$
Z(n)= \begin{cases}\frac{1}{2} & \text { if } A_{l}(n) \text { is }(r, E) \text {-singular } \\ \tilde{A}_{1} l^{s+1} e^{-r / n} & \text { if } A_{l}(n) \text { is regular }\end{cases}
$$

Suppose we encounter a singular box $N_{s}$ times. The maximum distance traveled in those $N_{s}$ steps is equal to the size of $J$ boxes, that is, $J\left(l+\frac{3}{2} \hat{l}+1\right)$. On the other hand, since $d\left(n_{0}, \partial \Lambda_{l}\left(n_{0}\right)\right)=\frac{1}{2}\left(L-L^{*}\right)=\frac{1}{2} \tilde{L}$, we can make at least $N_{r}=N-N_{s}$ regular steps, where

$$
N_{r} \frac{\hat{l}}{2}+J\left(l+\frac{3}{2} \hat{l}+1\right) \geqslant \frac{1}{2}(\tilde{L}-l)
$$

or

$$
N_{r} \geqslant \frac{\tilde{L}-J(2 l+3 \hat{l}+2)}{\hat{l}} \geqslant \frac{1}{2}\left(l^{x-1}-l^{x-1}-8 J-2 J l^{-1}\right)
$$


Therefore

$$
\begin{aligned}
Z\left(n_{0}\right) & \cdots Z\left(n_{N-1}\right) \\
& \leqslant\left(\tilde{A}_{1} l^{s+1} e^{-r r_{i n}}\right)^{N_{r}} \\
& \leqslant \exp \left\{\frac{l^{\alpha-1}}{2}\left[\ln \tilde{A}_{1}+(s+1) \ln l\right]-r l^{r_{0}}\left(\frac{l^{\alpha-1}}{2}-l^{\alpha s-1}-10 J\right)\right\}
\end{aligned}
$$

But $\gamma_{0}+\alpha-1>\alpha \gamma_{0}$, because $\gamma_{0}(\alpha-1)<\alpha-1$, since $\gamma_{0}<1$. We can therefore write

$$
Z\left(n_{0}\right) \cdots Z\left(n_{N-1}\right) \leqslant \frac{1}{2} e^{-r / r_{0 x}}=\frac{1}{2} e^{-r L i_{0}}
$$

The first term of (4.12) is bounded by

$$
2 \widetilde{C} e^{-(R / 4) / i s}<\frac{1}{2} e^{-r / 3 i)}
$$

for large $l$, since $\gamma_{0}<\gamma s$.

The next two lemmas are the analogues of Lemmas 7.5 and 7.1, respectively, in ref. 4 .

Lemma 4.7. Let $I \subset \mathbb{R}$ be an interval. Let $L_{0}>1$ and suppose that $K\left(L_{0}\right)$ holds. Let $l_{1}>l_{2} \geqslant l_{0}$ and let $A_{1}$ and $A_{l_{2}}$ be disjoint squares. Then

$$
\mathbb{P}\left\{d\left(\sigma^{\prime}\left(H_{A_{l_{1}}}\right), \sigma^{\prime}\left(H_{A_{l_{2}}}\right)\right)<e^{-l_{2}^{H_{2}}}\right\}<\frac{l_{1}^{2}}{l_{2}^{\prime}}
$$

where

$$
\sigma^{\prime}\left(H_{A_{l}}\right)=\sigma\left(H_{A_{i}}\right) \cap\left\{E: d(E, I)<\frac{1}{2} e^{-I^{\prime \prime}}\right\}
$$

Lemma 4.8. Let $1<\alpha<\min \left(2 s, \gamma_{0} s / \beta, p / 2\right)$. Then there exists $Q$ such that if $l>Q$ and $R(l, r)$ holds and $K(L)$ holds for all $L \geqslant l$, then $R(L, r)$ holds with $L=l^{\alpha}$.

Finally, we have the following result.

Theorem 4.9. Let $\alpha$ be as in the preceding lemma. Then there exists $Q_{0} \geqslant 1$ such that if for some $L_{0} \geqslant Q_{0}$, the conditions $R\left(L_{0}, r_{0}\right)$ and $K\left(L_{0}\right)$ hold, then $R\left(L_{k}, r_{0}\right)$ holds for all $k=0,1,2, \ldots$, where $L_{k+1}=L_{k}^{\alpha}$. 


\section{APPENDIX. CONVOLUTION ESTIMATES}

Here we give the proofs of the two technical Lemmas 2.1 and 3.3.

Proof of Lemma 2.1. We have

$$
s|z-n|^{2}+t\left|n-z^{\prime}\right|^{2}=(s+t)|n-\alpha|^{2}+\frac{s t}{s+t}\left|z-z^{\prime}\right|^{2}
$$

where $\alpha=\left(s z+t z^{\prime}\right) /(s+t)$. Therefore

$$
\begin{aligned}
& \sum_{n \in \mathbb{Z}[i]} \exp \left(-s|z-n|^{2}\right) \exp \left(-t\left|n-z^{\prime}\right|^{2}\right) \\
& \quad=\exp \left(-\frac{s t}{s+t}\left|z-z^{\prime}\right|^{2}\right) \sum_{n \in \mathbb{Z}[i]} \exp \left[-(s+t)|n-\alpha|^{2}\right]
\end{aligned}
$$

Now if $n_{1}-\alpha_{1} \geqslant 1$ and $n_{1}-1 \leqslant x \leqslant n_{1}$, then $\left(n_{1}-\alpha_{1}\right)^{2} \geqslant\left(x-\alpha_{1}\right)^{2}$. Therefore

$$
\begin{aligned}
\sum_{n_{1} \geqslant 1+x_{1}} e^{-(s+t)\left(n_{1}-x_{1}\right)^{2}} & \leqslant \sum_{n_{1} \geqslant 1+x_{1}} \int_{n_{1}-1}^{n_{1}} e^{-(s+t)\left(x-x_{1}\right)^{2}} d x \\
& \leqslant \int_{x_{1}}^{s_{0}} e^{-\left(s+()\left(x-x_{1}\right)^{2}\right.} d x=\frac{1}{2}\left(\frac{\pi}{s+t}\right)^{1 / 2}
\end{aligned}
$$

Similarly,

$$
\sum_{n_{1} \leqslant x_{1}-1} e^{-(s+t)\left(n_{1}-x_{1}\right)^{2}} \leqslant \frac{1}{2}\left(\frac{\pi}{s+t}\right)^{1 / 2}
$$

Thus

$$
\sum_{n_{1} \in \mathbb{Z}} e^{-(s+t)\left(n_{1}-x_{1}\right)^{2}} \leqslant 1+e^{-(s+t) / 4}+\left(\frac{\pi}{s+t}\right)^{1 / 2}
$$

and so

$$
\sum_{n \in \mathbb{Z}[i]} e^{-(x+1)|n-x|^{2}} \leqslant K(s+t)
$$

Proof of Lemma 3.3. We split the sum into three parts:

$$
\sum_{m \in \mathbb{Z}[i]} e^{-x\left\{|=-m| \dot{r}+\left|z^{\prime}-m\right|^{\mid}\right\}}=\mathrm{I}+\mathrm{II}+\mathrm{III}
$$


where

$$
\begin{aligned}
& \mathrm{I}=\sum_{\substack{|m-z| \geqslant 1 / 2\left|=-z^{\prime}\right| \\
\left|m-z^{\prime}\right| \geqslant|/ 2|=-z^{\prime} \mid}} e^{-x\left\{1=-m||^{2}+\left.\left|z^{\prime}-m\right|\right|^{\prime}\right\}} \\
& \mathrm{II}=\sum_{|m-z|<1 / 2\left|=-z^{\prime}\right|} e^{\left.-x\}\left.|=-m|\right|^{\prime}+\left.\left|z^{\prime}-m\right|\right|^{\prime}\right\}} \\
& \text { III }=\sum_{\left|m-z^{\prime}\right|<1 / 2\left|=-z^{\prime}\right|} e^{-x|| z-\left.m\right|^{i}+\left|z^{\prime}-m\right|^{\prime} !}
\end{aligned}
$$

If $|m-z| \geqslant \frac{1}{2}\left|z-z^{\prime}\right|$ and $\left|m-z^{\prime}\right| \geqslant \frac{1}{2}\left|z-z^{\prime}\right|$, then

$$
2^{r-1}\left\{|m-z|^{r}+\left|m-z^{\prime}\right|^{r}\right\} \geqslant\left|z-z^{\prime}\right|^{r}
$$

Therefore

$$
\mathrm{I} \leqslant e^{-x\left|=-z^{\prime}\right| ;} \sum_{m \in \mathbb{Z}[i]} e^{-x\left(1-2 i^{i-1}\right)|m-=| i}
$$

Now

$$
\sum_{m \in \mathbb{Z}[i]} e^{-x\left|1-2^{z-1}\right| m-z \mid z}
$$

is bounded by a constant $C_{1}(\gamma)$ independent of $z$ and thus

$$
\mathrm{I} \leqslant C_{1}(\gamma) e^{-x\left|=-z^{\prime}\right| \gamma}
$$

On the other hand, if $|m-z|<\frac{1}{2}\left|z-z^{\prime}\right|$, then

$$
\begin{aligned}
\left|m-z^{\prime}\right|^{\prime} & =\left|\left(z-z^{\prime}\right)-(z-m)\right|^{\prime} \\
& \geqslant\left(\left|z-z^{\prime}\right|-|z-m|\right)^{\gamma} \\
& =\left|z-z^{\prime}\right|^{\gamma}\left(1-\left|\frac{z-m}{z-z^{\prime}}\right|\right)^{\gamma} \\
& \geqslant\left|z-z^{\prime}\right|^{\gamma}\left(1-\left|\frac{z-m}{z-z^{\prime}}\right|\right) \\
& \geqslant\left|z-z^{\prime}\right|^{\gamma}\left(1-\frac{1}{2^{1-\gamma}}\left|\frac{z-m}{z-z^{\prime}}\right|^{\prime}\right) \\
& =\left|z-z^{\prime}\right|^{\gamma}-\frac{1}{2^{1-\gamma}}|z-m|^{\prime}
\end{aligned}
$$


Therefore

$$
\mathrm{II} \leqslant e^{-\alpha\left|z-z^{\prime}\right| \gamma} \sum_{m \in \mathbb{Z}[i]} e^{-x\left(1-1 / 2^{1-i}\right)|z-m|^{i}} \leqslant C_{2}(\gamma) e^{-x\left|=-z^{\prime}\right| i}
$$

Similarly,

$$
\mathrm{III} \leqslant C_{2}(\gamma) e^{-x\left|=-z^{\prime}\right| r}
$$

\section{ACKNOWLEDGMENTS}

We are grateful to $\mathrm{H}$. Kunz for suggesting this model and to L. A. Pastur for bringing ref. 7 to our attention. J.V.P. acknowledges support from the European Commission and would like to thank the Institut de physique théorique of the École Polytechnique Fédérale de Lausanne for their hospitality and financial support. T.C.D. and N.M. would like to thank University College, Dublin, for inviting them to Dublin, where this work was initiated. This work was partially supported by the European Commission under the Human Capital and Mobility Scheme (EU contract CHRX-CT93-0411).

\section{REFERENCES}

1. B. Huckestein, Rev. Mod. Phys. 67:357 (1995).

2. J. M. Combes and P. D. Hislop, Commun. Math. Phys. 177:603 (1996).

3. W.-M. Wang. Microlocalization, percolation and Anderson localization for the magnetic Schrödinger operator with a random potential, J. Funct. Anal., to appear.

4. T. C. Dorlas. N. Macris, and J. V. Pulé. Helv. Phys. Acta 68:330 (1995).

5. T. C. Dorlas, N. Macris, and J. V. Pulé, J. Math. Phys. 37:1574 (1996).

6. J. M. Barbaroux, J. M. Combes, and P. D. Hislop. Landau Hamiltonians with unbounded random potentials: Localization in unperturbed gaps, preprint (1996).

7. Y. Avishai, R. M. Redheffer, and Y. B. Band, J. Phys. A 25:3883 (1992); see also Y. Avishai and R. M. Redheffer, Phys. Rev. B 47:2089 (1993); Y. Avishai, M. Ya. Azbel', and S. A. Gredeskul, Phys. Rev. B 48:17280 (1993).

8. H. Kunz, Commun. Math. Phys. 112:121 (1987).

9. J. Bellisard, A. Van Elst, and H. Schulz-Baldes, J. Math. Phys. 35:5373 (1994).

10. D. J. Thouless, J. Phys. C 14:3475 (1981).

11. H. Aoki and T. Ando, J. Phys. Soc, Jpn. 54:2238 (1985).

12. H. Aoki and T. Ando, Phys. Rev. Letr. 54:831 (1985).

13. H. von Dreifus and A. Klein, Commun. Math. Phys. 124:285 (1989).

14. R. Carmona and J. Lacroix, Spectral Theory of Random Schrödinger Operators (Birkhauser, Boston, 1990).

15. R. Ph. Boas, Entire Functions (Academic Press, New York, 1954).

16. D. J. Thouless, J. Phys. C 17:L.325 (1984).

17. I. M. Gel'fand and N. Ya. Vilenkin, Generalized Functions, Vol. 4 (Academic Press, New York, 1964). 